\title{
Effect of Synthetic Jet with Duty Cycle over Heat Transfer Enhancement
}

\author{
Mehmet Seyhan $^{1}$, Hürrem Akbıyık ${ }^{2}$, Mustafa Sarığlu' ${ }^{1}$, Ersoy Fatih Erdurcan ${ }^{3}$, Yahya Erkan Akansu ${ }^{3}$ \\ ${ }^{1}$ Karadeniz Technical University \\ 61080, Trabzon, Turkey \\ mehmetseyhan@ktu.edu.tr; sarioglu@ktu.edu.tr \\ ${ }^{2}$ Çukurova University \\ 01170, Adana, Turkey \\ hakbiyik@cu.edu.tr \\ ${ }^{3}$ Niğde Ömer Halisdemir University \\ 51240, Niğde, Turkey \\ fatih.erdurcan@gmail.com; akansu@ohu.edu.tr
}

\begin{abstract}
Heat transfer performance of the synthetic jet actuator is experimentally investigated to determine the effect of duty cycles. Measurements are carried out at duty cycles of $20 \%, 40 \%, 60 \%, 80 \%$ and $100 \%$ (continuous signal) at $\mathrm{H} / \mathrm{D}=2,4$ and 6 . The Nusselt number distributions are obtained by using a thermal camera. The results of the heat transfer measurements indicate that the Nusselt number notably increases with increasing the duty cycle from $20 \%$ to $100 \%$ at $\mathrm{H} / \mathrm{D}=4$ and 6 . However, there is no such a behaviour in the case of $H / D=2$. It is shown that the increase in jet-to-heated surface distance (H/D) significantly changes the Nusselt number distribution.
\end{abstract}

Keywords: Duty Cycle, Synthetic Jet, Heat Transfer, Nusselt Number.

\section{Introduction}

Synthetic jets have been used for many years to improve heat transfer applications. The immense abilities of the SJs enable to solve problems in different applications such as flow control, cooling electronic devices, and improvement of fuel cell performance. This paper is concentrating on a cooling application with the synthetic jet and also with more emphasis on an experimental method. The synthetic jet actuation parameters can change the synthetic jet characteristics. Cooling efficiency is also dependent on these parameters. Rylatt and O'Donovan (2012) did an experimental study on the effect of a synthetic jet on heat transfer at $\mathrm{Re}=3000$ and $\mathrm{H} / \mathrm{D}=1$. They showed that the heat transfer ratio is directly related to the excitation frequency. Also, they showed that the interaction between the two jets is related to the deterioration of the coherent structure in the jet flow [1]. Duan and Wang reported that the increase in duty cycle factor affects the vortex ring acceleration and vortex rings move a longer distance from the orifice [2]. Heat transfer on the surface is increased by the impingement of synthetic jet flow on the heated surface. In order to increase the momentum transfer from the heated surface, the distance between the orifice of the synthetic jet and plate can be changed. McGuinn et al. (2013) investigated the impact of synthetic jet flow field and surface heat transfer distribution depending on stroke length. Experimental measurements were made by using a fast PIV and a single point hot wire anemometer with a stroke range of $3<\mathrm{L} / \mathrm{d}<32$ and a nozzle surface distance of $2<\mathrm{H} / \mathrm{D}<16$. Four different synthetic flow regimes have been defined depending on the threshold values for the stroke length (L/d). As a result, different multiplier jet flow regimes and heat transfer regimes can be optimized by adjusting the stroke length [3].In the experimental study of Liu et al. 2015, the flow and heat transfer characteristics are investigated in terms of actuation frequency, jet Reynolds number and jet-to-surface spacing. They reported that the highest heat transfer was achieved by using a jet-to-surface spacing of 15 [4]. Valiorgueet al. (2009) investigated the relation between the convective heat transfer characteristics and the impinging synthetic jet flow structure for a small jet to surface spacing $(\mathrm{H} / \mathrm{D}=2)$. They reported that the flow regime can be determined by the ratio of jet stroke length to surface spacing [5]. Chaudhari et al. 2009 studied the impinging synthetic jet to determine heat transfer 
characteristics for a longer distance between the synthetic jet and heated plate (0-25). They reported that $\mathrm{Nu}$ is increasing for the distance of 6 . However, when the distance is increased more than $6, \mathrm{Nu}$ is gradually decreasing[6].

Main purpose of this study is to investigate the effect of the synthetic jet driving by a duty cycle over the heat transfer enhancement for jet-to-heated surface distance.

\section{Experimental Setup}

The experimental setup of the synthetic jet actuator is depicted in Fig. 1. This setup consists of a synthetic jet actuator with speaker, a hot-wire probe, two axis traverse mechanisms, a computer, a function generator, an oscilloscope, a traverse controller, a hot wire anemometer, an amplifier, a power supply, a current probe and a BNC DAQ. The orifice diameter (D) is $15 \mathrm{~mm}$. The oscillation signal is generated from an AA TECH brand AWG-1010 function generator and then amplified before driving the speaker. This driving signal is monitored with an oscilloscope to eliminate the noise. A BOSS brand 1000W amplifier and JW-36 model $1000 \mathrm{~W}$ subwoofer loudspeaker are used. H indicates the distance between orifice surface and heated target plate. Velocity measurement is performed to obtain mean jet velocity with a 55P11 single wire probe at $\mathrm{H} / \mathrm{D}=2$.Data is collected via a NI PCI-6220 data acquisition card at a sampling frequency of $2 \mathrm{kHz}$ during 5 seconds.

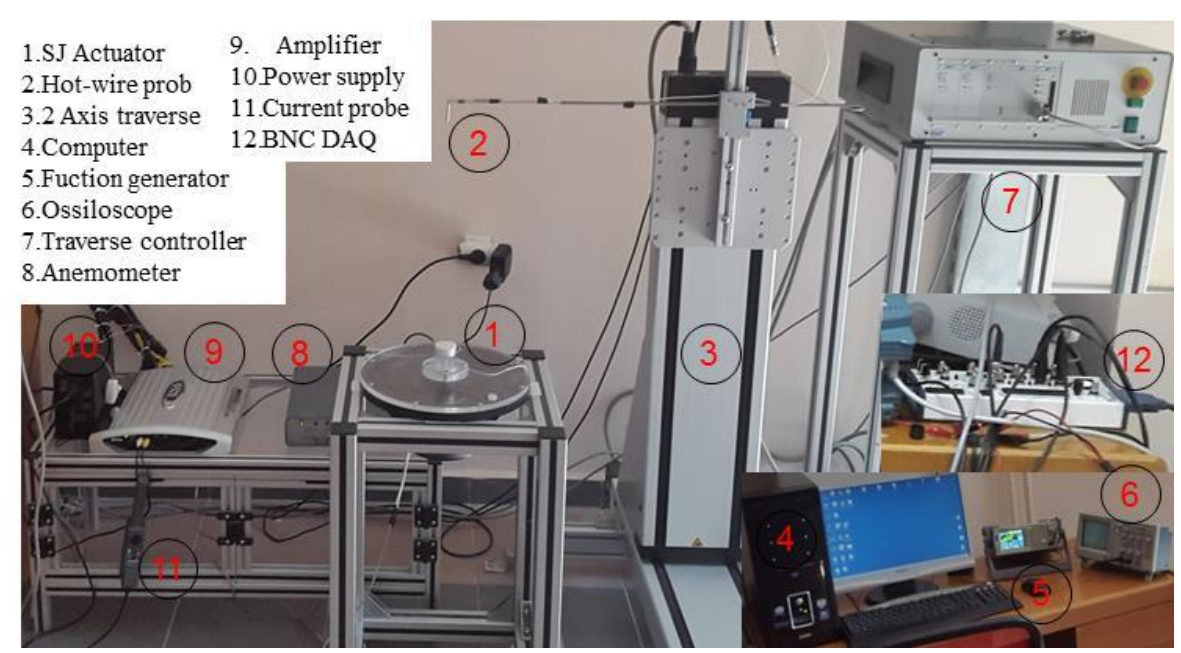

Fig. 1: Experimental setup of synthetic jet actuator for velocity measurements.

A schematic view of the heat transfer test setup is presented in Fig. 2. This setup is composed of a speaker, a thermal camera, a computer, a two axis traverse mechanism, a heated target plate, insulation and a power supply. The synthetic jet actuator is placed perpendicular to the heated target plate. The target plate having constant heat flux boundary condition is consists of a surface area of $240 \mathrm{~mm}$ x $240 \mathrm{~mm}$. Copper plate is insulated by Styrofoam and is also heated with a thin film heater. The temperatures of the Copper plate, the jet and ambient temperature is measured using thermocouples. Temperature distribution on the surface of the heated target plate is measured by a Testo 885-2 thermal camera. The plate is coated with a matt black colour in which case the value of the surface emissivity is 0.97 [1]. Heat transfer measurements are taken until reaching a steady state condition with a working synthetic jet actuator.

The actuator is driven by a sinusoidal signal having a driving frequency of $10 \mathrm{~Hz}$. Heat transfer experiments are performed for duty cycles (DC) of 20\%, 40\%, 60\%, $80 \%$ and $100 \%$ (continuous signal) at $\mathrm{H} / \mathrm{D}=2,4$ and 6 . Reynolds numbers are 1070 for $\mathrm{DC}=20 \%, 2040$ for $\mathrm{DC}=40 \%, 3000$ for $\mathrm{DC}=60 \%, 4000$ for $\mathrm{DC}=80 \%$ and 4750 for $\mathrm{DC}=100 \%$. 


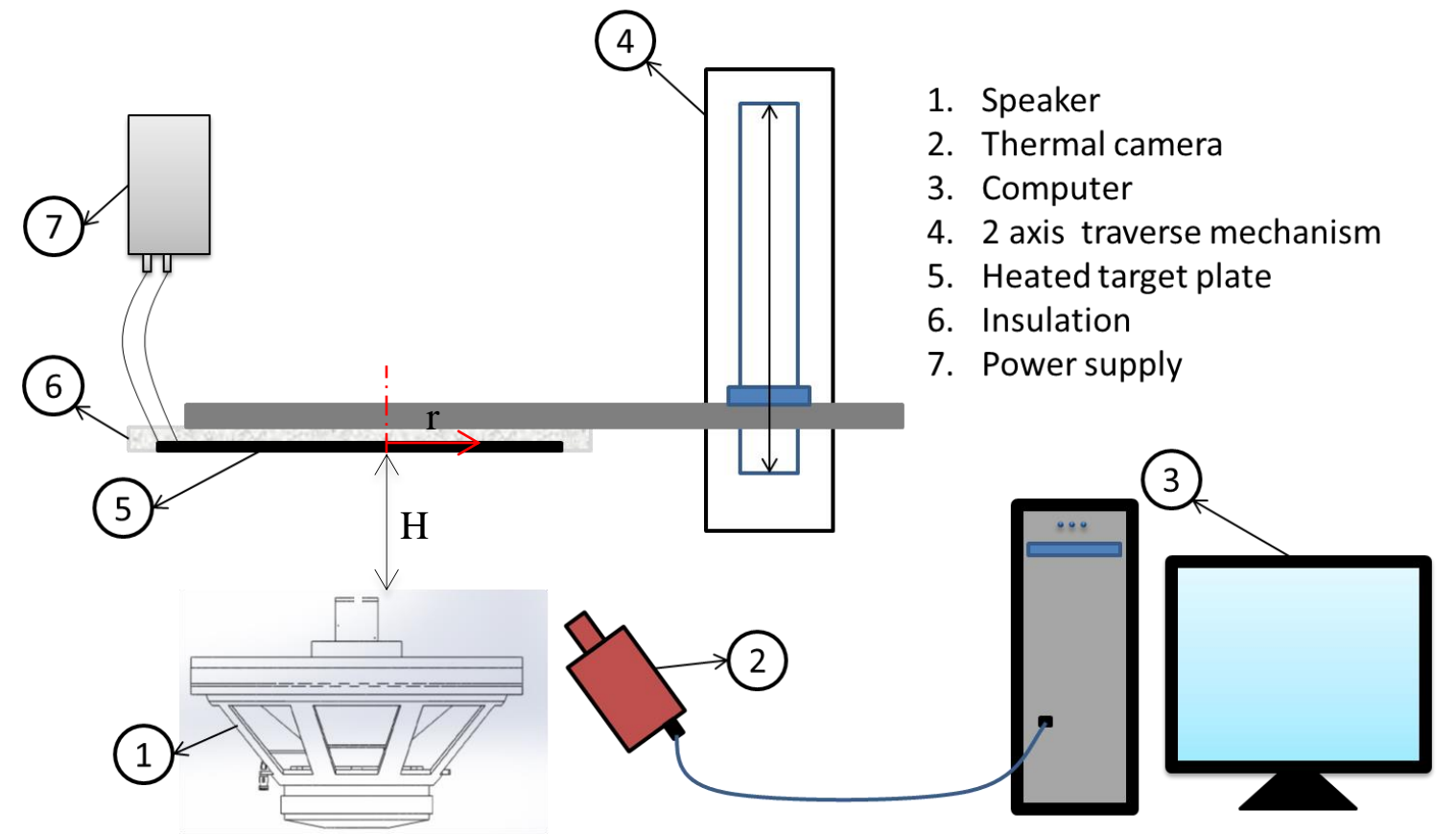

Fig. 2: Experimental setup of heat transfer measurement .

Data acquired from the heat transfer measurements are presented in the form of a time-averaged Nusselt number (Nu). The Nusselt number is calculated by using equations (1), (2) and (3). The heat flux, $\mathrm{q}_{\text {gen }}$ is calculated by;

$$
q_{g e n}^{\prime \prime}=\frac{\text { V.I }}{A_{\text {surf }}}
$$

Where, $\mathrm{V}$ is the supply voltage of the film heater, I is the supply current of the film heater and $\mathrm{A}_{\text {surf }}$ is the surface area of the heated copper plate. Supply voltage and current is adjusted using an adjustable DC power supply. Heat loss by radiation is neglected from calculations $[7,8]$. The heat transfer coefficient is found by:

$$
h=\frac{q_{g e n}^{\prime \prime}}{\left(T_{\text {surf }}-T_{j e t}\right)}
$$

where, $\mathrm{T}_{\text {surf }}$ is the surface temperature of the heated copper plate and $\mathrm{T}_{\mathrm{jet}}$ is the temperature of the synthetic jet. These temperatures are measured with the thermocouples. Finally the Nusselt number can be calculated with:

$$
N u=\frac{h \cdot D}{k}
$$

where, $\mathrm{D}$ is the diameter of the orifice and $\mathrm{k}$ is thermal conductivity.

\section{Results}

Synthetic jet driving signals for duty cycles (DC) of 20\%, 40\%, 60\%, $80 \%$ and $100 \%$ are presented in Fig 3(a). It can be seen that the number of waves increase with the duty cycle. While the duty cycle is $20 \%$, the number of waves is 2 . Fig. 3 (b) indicates the instantaneous velocity distribution for $\mathrm{DC}=20 \%, 40 \%, 60 \%, 80 \%$ and $100 \%$ at $\mathrm{H} / \mathrm{D}=2$. 

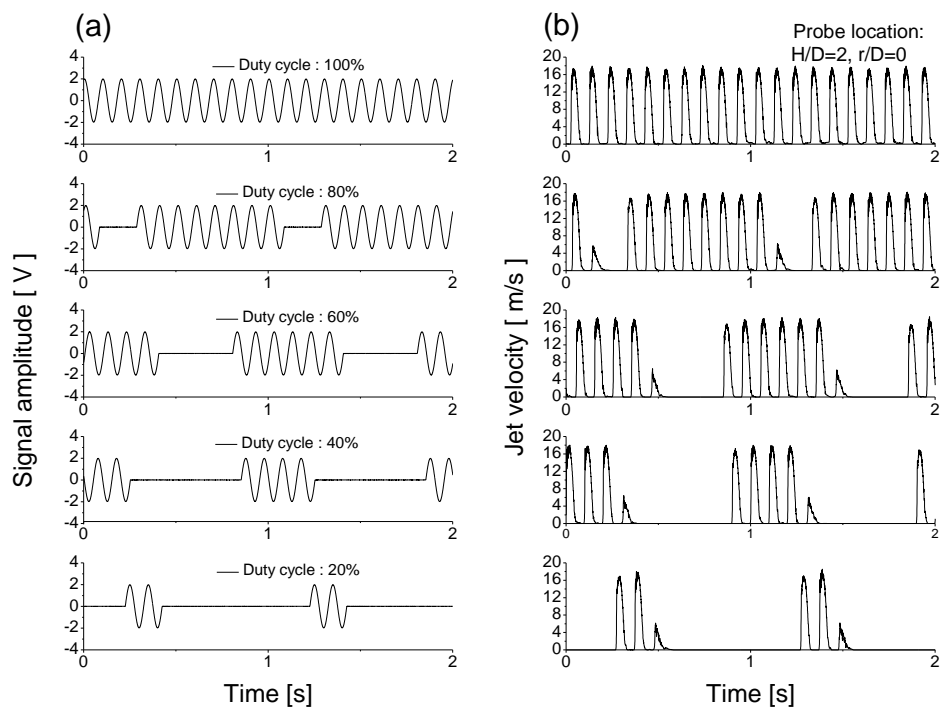

Fig. 3: (a) Duty cycle driving signal and (b) instantaneous velocity distribution for duty cycle (DC) of 20\%, 40\%, 60\%, $80 \%$ and $100 \%$ at $\mathrm{H} / \mathrm{D}=2$.

The time-averaged Nusselt number distributions as a function of r/D are presented in Fig. 4 for the duty cycle of $20 \%$, $40 \%, 60 \%, 80 \%$ and $100 \%$ at $\mathrm{H} / \mathrm{D}=2$. At the stagnation region, heat transfer provided by the synthetic jet has the maximum value. When radial distance on the heated plate surface is increased, heat transfer is notably decreased from r/D $=0$ to $\mathrm{r} / \mathrm{D}=7$ as can be seen in Fig. 4, 5 and 6 . Fig 4 clearly shows the fall in Nu number with a decreasing duty cycle. This is attributed to the number of jets being proportional to the duty cycle. Nusselt number distribution for DC $=80 \%$ is lower than that for $\mathrm{DC}=100 \%$ between $\mathrm{r} / \mathrm{D}=0$ and $\mathrm{r} / \mathrm{D}=1$ and a similar trend can be seen after $\mathrm{r} / \mathrm{D}=1$.

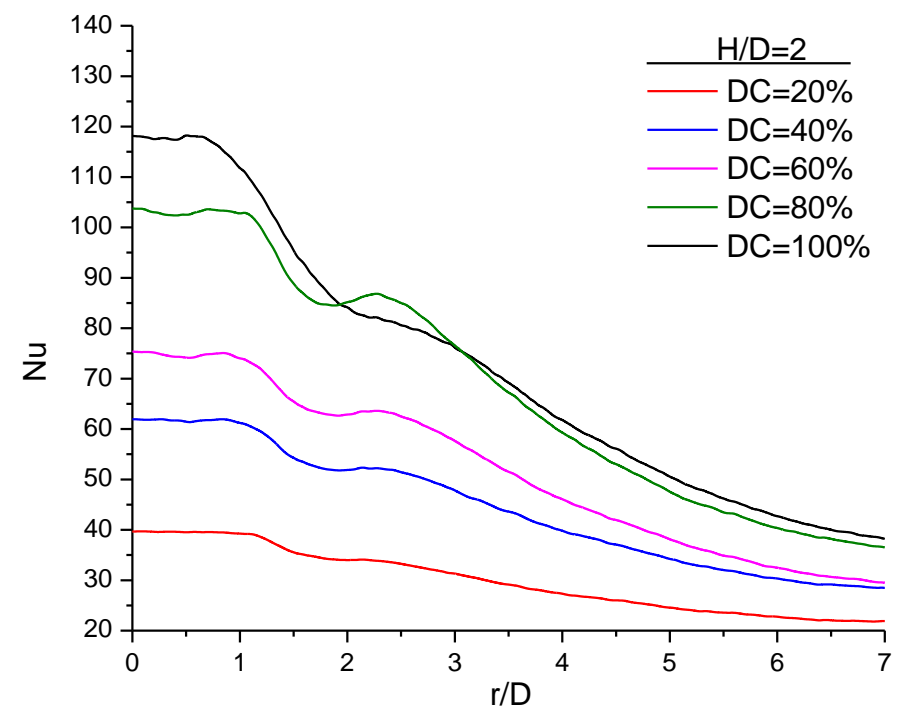

Fig. 4:Time-averaged Nusselt number versus r/D for duty cycle (DC) of 20\%, 40\%, 60\%, $80 \%$ and 100\%at H/D=2.

Fig. 5 shows the variation of the time-averaged Nusselt number with normalized radial distance (r/D) for duty cycles (DC) of $20 \%, 40 \%, 60 \%, 80 \%$ and $100 \%$ at $\mathrm{H} / \mathrm{D}=4$. Observations clearly show that the Nusselt number significantly increases with increasing duty cycle from $\mathrm{DC}=20 \%$ to $\mathrm{DC}=100$. The maximum Nusselt number corresponding to the stagnation point is found to be 132 at $\mathrm{DC}=100 \%$. The Nusselt number is almost constant between $\mathrm{r} / \mathrm{D}=0$ and $\mathrm{r} / \mathrm{D}=0.7$ for all duty cycle values and then starts to decrease significantly after $\mathrm{r} / \mathrm{D}=0.7$.The time-averaged Nusselt number 
distribution as a function of r/D is given in Fig. 6 for the $20 \%, 40 \%, 60 \%, 80 \%$ and $100 \%$ duty cycles at $\mathrm{H} / \mathrm{D}=6 . \mathrm{When}$ comparing Fig.6 to Fig 5. A similar trend in Nusselt number distribution with changing duty cycle percentage ratio can be seen. The maximum Nusselt number value is 136.5 at $\mathrm{DC}=100 \%$.

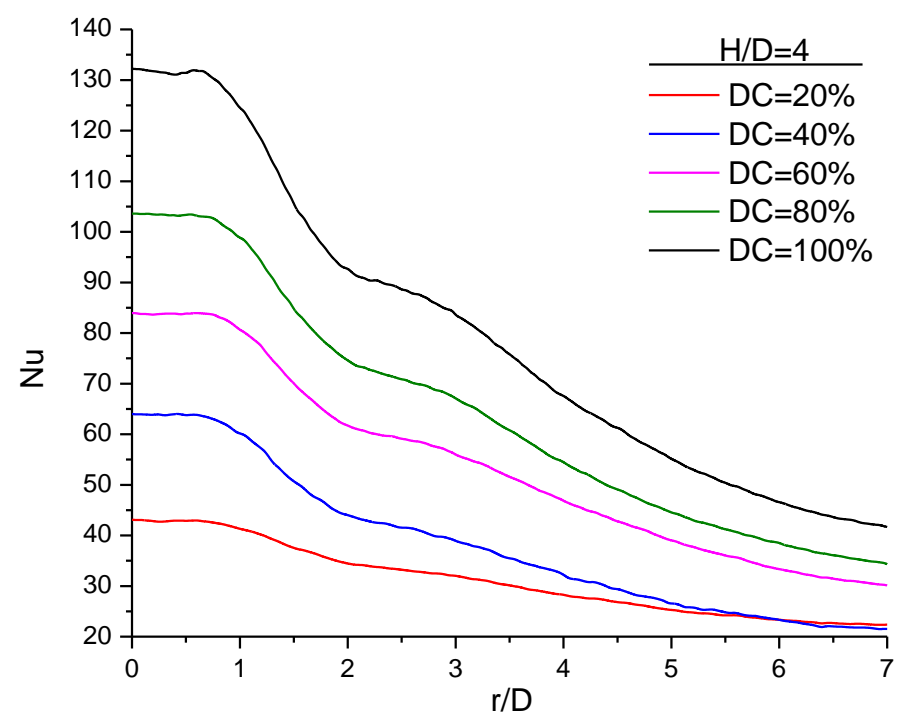

Fig. 5: Time-averaged Nusselt number versus r/D for duty cycle (DC) of 20\%, 40\%, 60\%, 80\% and 100\%at H/D= 4 .

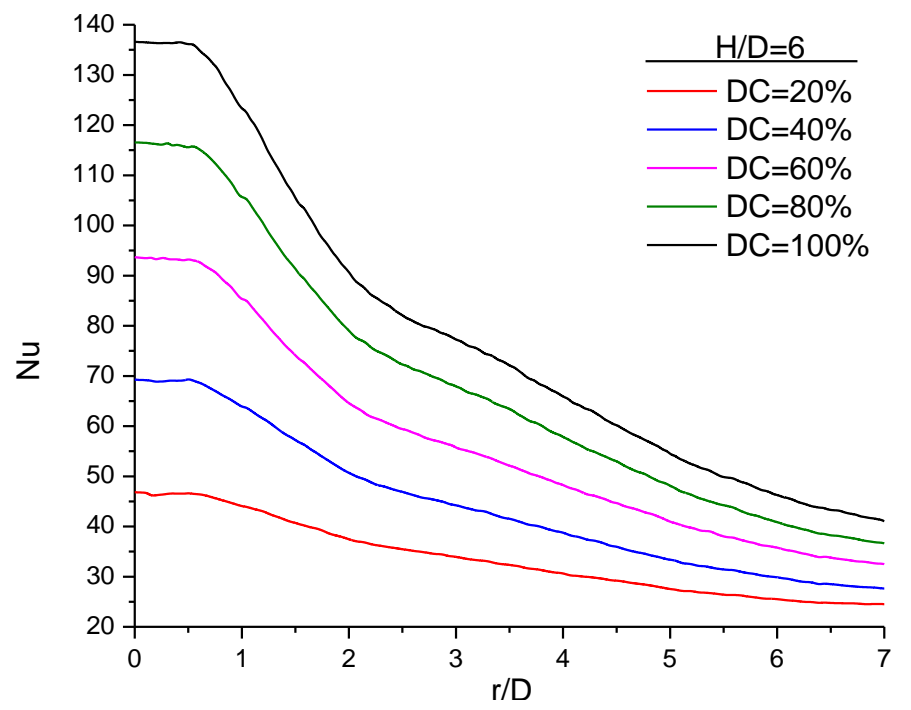

Fig. 6: Time-averaged Nusselt number versus r/D for duty cycle (DC) of 20\%, 40\%, 60\%, 80\% and 100\%at H/D=6.

The variations of time-averaged Nusselt number as a function of normalized radial distance at H/D $=2,4$ and 6 for DC $=20 \%, 40 \%, 60 \%, 80 \%$ and $100 \%$ are presented in Fig. 7. The Optimum jet-to-heated surface distance changes with increasing duty cycle value. For $\mathrm{DC}=20 \%$ and $60 \%, \mathrm{Nu}$ at stagnation region augments with increasing H/D. After $\mathrm{r} / \mathrm{D}=2$ for $\mathrm{DC}=60 \%$ and $100 \%$, Nusselt number distributions are almost independent of jet-to-heated surface distance (r/D). Optimum jet-to-heated surface distance at stagnation region is $\mathrm{H} / \mathrm{D}=6$ at all duty cycles. 


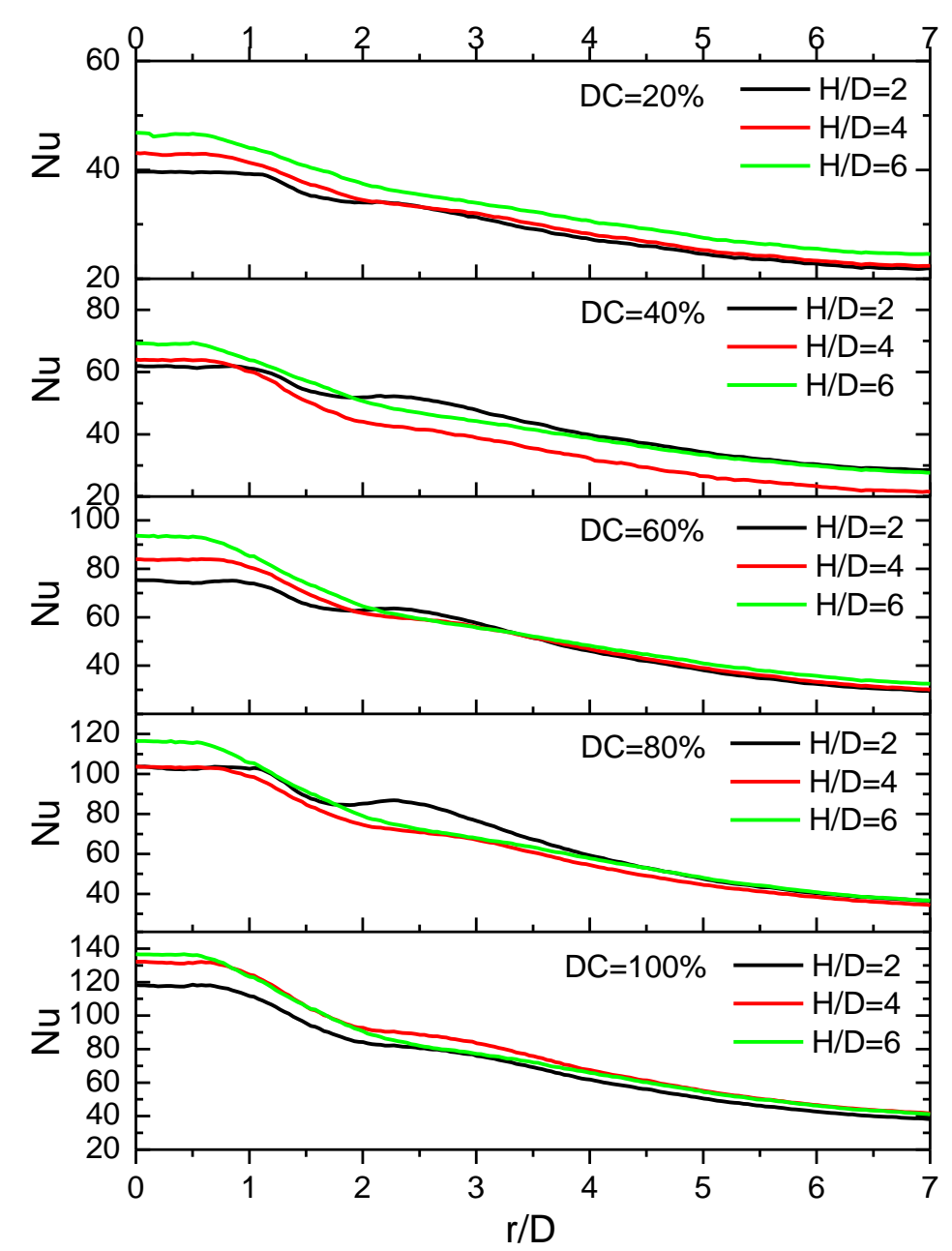

Fig. 7: Time-avareged Nusselt number versus $\mathrm{r} / \mathrm{D}$ at $\mathrm{H} / \mathrm{D}=2,4$ and 6 for $\mathrm{DC}=20 \%, 40 \%, 60 \%, 80 \%$, and $100 \%$.

\section{Conclusion}

In this paper, the heat transfer performance of a synthetic jet actuator with driven with different duty cycles is investigated. The effects of the duty cycle varying from $20 \%$ to $100 \%$ and jet-to-heated surface distance $(\mathrm{H} / \mathrm{D}=2,4$ and 6$)$ are explored as experimental parameters. The Nusselt number is obtained by measuring the temperature distribution with a thermal camera. The results show that the Nusselt number significantly changes with increasing duty cycle. At H/D = 4 and 6, Nusselt number distribution as a function of normalized radial distance augments with increasing duty cycle percentage ratios. The results indicate that there is a change in Nusselt number distribution with the variation of jet-toheated surface distance (H/D).

\section{Acknowledgements}

The authors would like to acknowledge the financial support of this work by the Scientific and Technological Research Council of Turkey (TUBITAK) under the Contract Number of 215M920.

\section{References}

[1] D. I. Rylatt, T. S. O’Donovan, "Heat transfer enhancement to a confined impinging synthetic air jet," Appl. Therm. Eng., vol. 51, pp. 468-475, 2013.

[2] T. Duan, J. Wang, "Experimental investigation on the evolution of axi-symmetrical synthetic jet," J. Vis., vol. 19, pp. 351-358, 2016. 
[3] A. McGuinn, R. Farrelly, T. Persoons, D. B. Murray, "Flow regime characterisation of an impinging axisymmetric synthetic jet," Exp. Therm. Fluid Sci.,vol. 47, pp. 241-251, 2013.

[4] Y. H. Liu, S. Y. Tsai, C. C. Wang, "Effect of driven frequency on flow and heat transfer of an impinging synthetic air jet," Appl. Therm. Eng., vol. 75, pp. 289-297, 2015.

[5] P. Valiorgue, T. Persoons, A. McGuinn, D. B. Murray, "Heat transfer mechanisms in an impinging synthetic jet for a small jet-to-surface spacing," Exp. Therm. Fluid Sci., vol. 33, pp. 597-603, 2009.

[6] M. Chaudhari, B. Puranik, A. Agrawal, "Heat transfer characteristics of synthetic jet impingement cooling," Int. J. Heat Mass Transf., vol. 53, pp. 1057-1069, 2010.

[7] Y. H. Liu, T. H. Chang, C. C. Wang, "Heat transfer enhancement of an impinging synthetic air jet using diffusionshaped orifice," Appl. Therm. Eng., vol. 94, pp. 178-185, 2016.

[8] J. Zhang, X. Tan, "Experimental study on flow and heat transfer characteristics of synthetic jet driven by piezoelectric actuator," Sci. China, Ser. E Technol. Sci., vol. 50, pp. 221-229, 2007. 